\title{
Quasi-permutable normal operators in octonion Hilbert spaces and spectra
}

\author{
Ludkovsky S.V.
}

15 March 2012

\begin{abstract}
Families of quasi-permutable normal operators in octonion Hilbert spaces are investigated. Their spectra are studied. Multiparameter semigroups of such operators are considered. A non-associative analog of Stone's theorem is proved. 1
\end{abstract}

\section{Introduction}

The theory of bounded and unbounded normal operators over the complex field is classical and have found many-sided applications in functional analysis, differential and partial differential equations and their applications in the sciences [4, 11, 12, 14, 32]. Nevertheless, hypercomplex analysis is fast developing, because it is closely related with problems of theoretical and mathematical physics and of partial differential equations [2, 7, 9]. On the other hand, the octonion algebra is the largest division real algebra in which the complex field has non-central embeddings [3, 1, 13]. The octonion algebra also is intensively used in mathematics and various applications [5, 10, 8, 15, 16].

\footnotetext{
${ }^{1}$ key words and phrases: non-commutative functional analysis, hypercomplex numbers, quaternion skew field, octonion algebra, quasi-permutable operators, spectra, spectral measure, non-commutative integration Mathematics Subject Classification 2010: 30G35, 17A05, 17A70, 47A10, 47L30, 47L60
} 
Previously analysis over quaternion and octonions was developed and spectral theory of bounded normal operators and unbounded self-adjoint operators was described [18, 19, 20, 21, 22]. Their applications in partial differential equations were outlined [23, 24, 25, 26, 27]. This paper is devoted to families of quasi-permutable normal operators in octonion Hilbert spaces. Their spectra are studied. Multiparameter semigroups of such operators are considered. A non-associative analog of Stone's theorem is proved.

Notations and definitions of papers [18, 19, 20, 21, 22] are used below. The main results of this article are obtained for the first time.

\section{Quasi-permutability of normal operators}

1. Definitions. If ${ }_{j} A$ is a set of $\mathbf{R}$ homogeneous $\mathcal{A}_{v}$ additive operators with $\mathcal{A}_{v}$ vector domains $\mathcal{D}\left({ }_{j} A\right)$ dense in a Hilbert space $X$ over the Cayley-Dickson algebra $\mathcal{A}_{v}, 2 \leq v, j \in \Lambda, \Lambda$ is a set, then we denote by $\operatorname{alg}_{\mathcal{A}_{v}}\left({ }_{j} A: j \in \Lambda\right)$ a family of all operators $B$ with $\mathcal{A}_{v}$ vector domains in $X$ obtained from $\left({ }_{j} A: j \in \Lambda\right)$ by a finite number of operator addition, operator multiplication and left and right multiplication of operators on Cayley-Dickson numbers $b \in \mathcal{A}_{v}$ or on $b I$, where $I$ denotes the unit operator on $X$.

Let ${ }_{1} A$ and ${ }_{2} A$ be two normal operators in a Hilbert space $X$ over the Cayley-Dickson algebra $\mathcal{A}_{v}, 2 \leq v$. Suppose that ${ }_{1} A$ and ${ }_{2} A$ are affiliated with a quasi-commutative von Neumann algebra A over $\mathcal{A}_{v}$ with $2 \leq v \leq 3$. Let ${ }_{1} E$ and ${ }_{2} E$ be their $\mathcal{A}_{v}$ graded projection valued measures defined on the Borel $\sigma$-algebra of subsets in $\mathcal{A}_{v}$ (see also $\S 2$ and $\S \S I .2 .58$ and I.2.73 in [28]). In this section the simplified notation $E$ instead of $\hat{\mathbf{E}}$ will be used.

We shall say that two normal operators ${ }_{1} A$ and ${ }_{2} A$ quasi-permute if

$$
\text { (1) }{ }_{1} E\left(\delta_{1}\right){ }_{2} E\left(\delta_{2}\right)={ }_{2} E\left(\delta_{2}\right){ }_{1} E\left(\delta_{1}\right)
$$

for each Borel subsets $\delta_{1}$ and $\delta_{2}$ in $\mathcal{A}_{v}$.

Operators $A,{ }_{1} A$ and ${ }_{2} A$ are said to have property $P$ if they satisfy the following four conditions $(P 1-P 4)$ :

$(P 1)$ they are normal, 
$(P 2)$ they are affiliated with a von Neumann algebra $A$ over either the quaternion skew field or the octonion algebra $\mathcal{A}_{v}$ with $2 \leq v \leq 3$ and

$$
\text { (P3) } \quad A={ }_{1} A{ }_{2} A \text { and }
$$

$(P 4)$ the family $\operatorname{alg}_{\mathcal{A}_{v}}\left(I, A, A^{*},{ }_{1} A,{ }_{1} A^{*},{ }_{2} A,{ }_{2} A^{*}\right)=: \mathrm{Q}\left(A,{ }_{1} A,{ }_{2} A\right)=: \mathrm{Q}$ over $\mathcal{A}_{v}$ generated by these three operators is quasi-commutative, that is a von Neumann algebra

$$
\operatorname{cl}\left[\operatorname{alg}_{\mathcal{A}_{v}}\left(I, A E(\delta), A^{*} E(\delta),{ }_{1} A{ }_{1} E\left(\delta_{1}\right),{ }_{1} A^{*}{ }_{1} E\left(\delta_{1}\right),{ }_{2} A_{2} E\left(\delta_{2}\right),{ }_{2} A^{*}{ }_{2} E\left(\delta_{2}\right)\right)\right] \subset
$$
$L_{q}(X)$

contained in $L_{q}(X)$ is quasi-commutative for each bounded Borel subsets $\delta$, $\delta_{1}, \delta_{2} \in \mathcal{B}\left(\mathcal{A}_{r}\right)$, where $2 \leq v \leq 3$.

It is possible to consider a common domain $\mathcal{D}^{\infty}(\mathrm{Q}):=\cap_{T \in \mathrm{Q}} \mathcal{D}^{\infty}(T)$ for a family of operators $\mathrm{Q}$, where $\mathcal{D}^{\infty}(T):=\cap_{n=1}^{\infty} \mathcal{D}\left(T^{n}\right)$. Then the family $\mathrm{Q}$ on $\mathcal{D}^{\infty}(\mathrm{Q})$ can be considered as an $\mathcal{A}_{v}$ vector space. Take the decomposition $\mathrm{Q}=\mathrm{Q}_{0} i_{0} \oplus \mathrm{Q}_{1} i_{1} \oplus \ldots \oplus \mathrm{Q}_{2^{v}-1} i_{2^{v}-1}$ of this $\mathcal{A}_{v}$ vector space with pairwise isomorphic real vector spaces $Q_{0}, Q_{1}, \ldots, Q_{2^{v}-1}$. Then as in $\S 2.5$ [29] for each operator $B \in \mathrm{Q}$ we put

$$
\text { (2) } B=\sum_{j}^{j} B \text { with }{ }^{j} B=\hat{\pi}^{j}(B) \in \mathrm{Q}_{j} i_{j}
$$

for each $j$, where $\hat{\pi}^{j}: \mathbf{Q} \rightarrow \mathbf{Q}_{j} i_{j}$ is the natural $\mathbf{R}$ linear projection, real linear spaces $\mathrm{Q}_{j} i_{j}$ and $i_{j} \mathrm{Q}_{j}$ are considered as isomorphic, so that

$$
\text { (3) } \sum_{k=0}^{2^{v}-1}{ }^{k} \hat{T}=T \text {. }
$$

If $E$ is an $\mathcal{A}_{v}$ graded projection valued measure on the Borel $\sigma$-algebra $\mathcal{B}\left(\mathcal{A}_{v}\right)$ for a normal operator $T \in \mathrm{Q}$, for uniformity of this notation we put also

(4) ${ }^{k} \hat{E}(d z) \cdot t y=\hat{\pi}^{k} E(d z) \cdot t y$

for every vector $y \in X$ and $t=t_{0} i_{0}+\ldots+t_{2^{v}-1} i_{2^{v}-1} \in \mathcal{A}_{v}$, where $z \in \mathcal{A}_{v}$, $t_{0}, \ldots, t_{2^{v}-1} \in \mathbf{R}, \quad E(d z) . t y=E(d z) .(t y)$.

2. Lemma. Let operators $A, B$ and $D$ have property $P$ and let $F$ be an $\mathcal{A}_{v}$ graded projection operator which quasi-permutes with $A$ so that $\mathcal{R}(F) \subset \mathcal{D}(A)$, where $\mathcal{D}(A)=\operatorname{Domain}(A), \quad \mathcal{R}(A)=$ Range $(A)$. Suppose that $G, H$ and $J$ are the restrictions of $A, F B$ and $F D$ to $\mathcal{R}(F)$ respectively. 
Then $G, H$ and $J$ are bounded operators so that $H$ and $J$ quasi-permute with $G$. Moreover, $H^{*}$ and $J^{*}$ are the restrictions to $\mathcal{R}(F)$ of $B^{*} F^{*}$ and $C^{*} F^{*}$ respectively, where

(1) ${ }^{j}\left(\hat{B}^{*}\right)^{k}\left(\hat{F}^{*}\right)=(-1)^{\kappa(j, k)+\eta(k) k} \hat{F}^{j}\left(\hat{B}^{*}\right)$ and

$(2)^{j}\left(\hat{D}^{*}\right)^{k}\left(\hat{F}^{*}\right)=(-1)^{\kappa(j, k)+\eta(k) k} \hat{F}^{j}\left(\hat{D}^{*}\right)$

for each $j, k$, with $\kappa(j, k)=0$ for $j=k$ or $j=0$ or $k=0, \kappa(j, k)=1$ for $j \neq k \geq 1, \quad \eta(0)=0, \quad \eta(k)=1$ for each $k \geq 1$.

Proof. Note 2.5 and Theorems 2.29, 2.44 and Proposition 2.32 in [29] and Definitions 1 imply that in components the following formulas are satisfied:

(3) ${ }_{1}^{j} \hat{E}\left(\delta_{1}\right){ }_{2}^{k} \hat{E}\left(\delta_{2}\right)=(-1)^{\kappa(j, k)}{ }_{2}^{k} \hat{E}\left(\delta_{2}\right)_{1}^{j} \hat{E}\left(\delta_{1}\right)={ }_{2}^{j} \hat{E}\left(\delta_{2}\right){ }_{1}^{k} \hat{E}\left(\delta_{1}\right)$

for each $j, k=0,1,2, \ldots$, where $\kappa(j, k)=0$ for $j=k$ or $j=0$ or $k=0$, $\kappa(j, k)=1$ for $j \neq k \geq 1$,

where $\theta_{k}^{j}\left(x_{j}\right)$ is denoted by $x_{j}$ for short, $\theta_{k}^{j}: X_{j} \rightarrow X_{k}$ is an R-linear topological isomorphism of real normed spaces (see $\S \S I .2 .1$ and I.2.73 in [28]). Suppose that $x, y \in \mathcal{R}(F)$, hence $x, y \in \mathcal{D}(B)=\mathcal{D}\left(B^{*}\right)$, since $\mathcal{R}(F) \subset \mathcal{D}(A) \subset \mathcal{D}(B)$. Therefore

(4) $<F B x ; y>=<B x ; y>=<x ; B^{*} y>=<x ; B^{*} y>=<x ; F B^{*} y>$ and $(5)<{ }^{j} \hat{F}^{k} \hat{B} x_{k} ; y_{j}>=<{ }^{k} \hat{B} x_{k} ; y_{j}>i_{j}^{*}=(-1)^{\kappa(j, k)+\eta(k)}<x_{k} ;{ }^{j} \hat{F}^{k}\left(\hat{B}^{*}\right) y_{j}>$. If $L=\left.F B^{*}\right|_{\mathcal{R}(F)}$, then $H^{*}=L$ and $H=L^{*}$ by Formula (4). The operator $L^{*}$ is closed, consequently, $H$ is closed and $\mathcal{D}(H) \supset \mathcal{R}(F)$. In view of the closed graph theorem for $\mathbf{R}$-linear operators the operator $H$ is bounded 1.8.6 [12]. This implies that the operator $G$ is also bounded, since the operator $A$ is normal and hence closed so that $\mathcal{R}(F) \subset \mathcal{D}(A)$. In view of Theorems $2.27,2.29$ an 2.44 in [29] the operator $A$ has an $\mathcal{A}_{v}$ graded projection valued measure. Take now $x \in \mathcal{R}(F)$, hence $A x \in \mathcal{R}(F) \subset \mathcal{D}(A) \subset \mathcal{D}(B)$, since

$$
{ }^{j} \hat{F}^{k} \hat{F}=(-1)^{\kappa(j, k) k} \hat{F}^{j} \hat{F} \text { and } \mathcal{D}(F)=\mathcal{D}(F)_{0} i_{0} \oplus \ldots \oplus \mathcal{D}(F)_{m} i_{m} \oplus \ldots
$$

for each $j, k$ and

$$
A=\int_{\mathcal{A}_{v}} F(d t) \cdot t
$$

so that ${ }^{j} \hat{F}^{k} \hat{A} \subseteq(-1)^{\kappa(j, k) k} \hat{A}^{j} \hat{F}$ for each $j, k$. Symmetric proof is for $A$ and $C$ instead of $A$ and $B$. The operators $B^{*} B$ and $C^{*} C$ belong to the family $\operatorname{alg}_{\mathcal{A}_{v}}\left(I, A, A^{*}, B, B^{*}, C, C^{*}\right)$. 
In view of Theorem I.3.23 [28] the spectra of $B^{*} B=\int_{-\infty}^{\infty} B^{*} B F(d t) \cdot t^{2}$ and $D^{*} D=\int_{-\infty}^{\infty} D^{*} D F(d t) \cdot t^{2}$ are real so that ${ }_{B^{*} B} F$ and ${ }_{D^{*} D} F$ are $\mathcal{A}_{v}$ graded projection valued measures for $B^{*} B$ and $D^{*} D$ respectively on $\mathcal{B}(\mathbf{R}) \subset \mathcal{B}\left(\mathcal{A}_{r}\right)$. Then from Formulas $(2,4)$ and $1(1, P 1-P 4)$ we deduce that

$$
\begin{aligned}
& (7)\left({ }^{j} \hat{F}^{k} \hat{B}\right){ }^{s} \hat{A} x_{s}=\left({ }^{j} \hat{F}^{k} \hat{B}\right) \sum_{p, q: i_{p} i_{q}=i_{s}}\left[{ }^{p} \hat{D}{ }^{q} \hat{B}+(-1)^{\kappa(p, q){ }^{q}} \hat{D}^{p} \hat{B}\right] \\
= & \sum_{p, q: i_{p} i_{q}=i_{s}}\left[\left({ }^{j} \hat{F}{ }^{k} \hat{B}\right)\left({ }^{p} \hat{D}{ }^{q} \hat{B}\right)+(-1)^{\kappa(p, q)}\left({ }^{j} \hat{F}^{k} \hat{B}\right)\left({ }^{q} \hat{D}{ }^{p} \hat{B}\right)\right] \\
= & (-1)^{\kappa(s, l) s} \hat{A}\left({ }^{j} \hat{F}^{k} \hat{B} x_{s}\right),
\end{aligned}
$$

since the set theoretic composition of operators is associative: $(F B)(D B)=$ $F((B D) B)$, where $l$ is such that $i_{j} i_{k} \in \mathbf{R} i_{l}$. Thus $H$ and analogously $J$ quasi-permute with $G$, since the family $\operatorname{alg}_{\mathcal{A}_{v}}\left(I, A, A^{*},{ }_{1} A,{ }_{1} A^{*},{ }_{2} A,{ }_{2} A\right)$ is quasi-commutative. From Formulas $(5,6)$ we infer Equalities $(1,2)$.

3. Notation. Suppose that $a, b \in \mathcal{A}_{r}$. If $b_{j} \geq a_{j}$ for each $j=$ $0,1,2, \ldots, 2^{r}-1$, this fact will be denoted by $b \succeq a$. Then $\mathcal{I}_{a, b}:=\{z \in$ $\left.\mathcal{A}_{r}: b \succeq z \succeq a\right\}$.

4. Lemma. Let operators $A, B$ and $D$ have property $P$ and let $F$ be an $\mathcal{A}_{v}$ graded projection valued measure for $A$, let also $b \succeq a \in \mathcal{A}_{v}$. Then $\mathcal{R}\left(F\left(\mathcal{I}_{a, b}\right)\right)=: Y$ reduces both $B$ and $D$ and these operators restricted to $Y$ are bounded and normal and they quasi-permute with the restriction $\left.A\right|_{Y}$.

Proof. Consider the pair of operators $A$ and $B$. Put ${ }_{n} F:=\left.F\right|_{\mathcal{I}_{-b(n), b(n)}}$ and ${ }_{n} V=\mathcal{R}\left(F\left(\mathcal{I}_{-b(n), b(n)}\right)\right)$ with $b(n)_{j}=n i_{j}$ for every $n \in \mathbf{N}$ and each $j=0,1,2, \ldots, 2^{v}-1$. Then ${ }_{n} V \subset{ }_{n+1} V$ for each $n$. Therefore, an $\mathcal{A}_{v}$ vector subspace $\bigcup_{n}{ }_{n} V=: V$ is dense in the Hilbert space $X$ over the CayleyDickson algebra $\mathcal{A}_{v}$, consequently, $\lim _{n}{ }_{n} F=I$ in the strong operator topology. Each operator ${ }_{n} A:=\left.A\right|_{n}$ is bounded and normal and has the $\mathcal{A}_{v}$ graded projection valued measure on the Borel $\sigma$-algebra $\mathcal{B}\left(\mathcal{A}_{v}\right)$ of all Borel subsets in $\mathcal{A}_{v}$ so that ${ }_{n} F=\left.F\right|_{n}$ for each natural number $n$. We consider the restriction ${ }_{n} G:=\left.{ }_{n} F B\right|_{n} V$. It is known from Lemma 2, that each operator ${ }_{n} G$ is bounded and quasi-permutes with ${ }_{n} B$ so that

$$
\text { (1) }{ }_{n^{G}}{ }^{j} \hat{F}_{n^{B}}^{k} \hat{F}=(-1)^{\kappa(j, k)}{ }_{n^{B}}^{k} \hat{F}_{n^{G}}^{j} \hat{F}
$$

for each $j, k$, consequently,

$$
\text { (2) } \quad{ }_{n}^{s} \hat{F}(\delta)\left({ }_{n^{B}}^{j} \hat{F}\left(\delta_{1}\right){ }_{n}^{k} \hat{F}\left(\delta_{2}\right) x\right)=(-1)^{\kappa(j, k)}{ }_{n}^{s} \hat{F}(\delta)\left({ }_{n}^{k} \hat{F}\left(\delta_{2}\right){ }_{n^{B}}^{j} \hat{F}\left(\delta_{1}\right) x\right)
$$


for each $x \in{ }_{n} V_{0}$ and $\delta, \delta_{1}, \delta_{2} \in \mathcal{B}\left(\mathcal{A}_{v}\right)$, where ${ }_{n} G F$ and ${ }_{n^{B}} F$ denote $\mathcal{A}_{v}$ graded projection valued measures for the operators ${ }_{n} G$ and ${ }_{n} B$ correspondingly.

Let now $y \in \mathcal{D}(A)_{0}$ and $\delta \in \mathcal{B}\left(\mathcal{A}_{v}\right)$ be fixed, hence

$$
\begin{gathered}
\text { (3) } \lim _{n}{ }_{n}^{s} \hat{F}(\delta)\left({ }_{n}^{k} \hat{F}\left(\delta_{2}\right)_{n^{B}}^{j} \hat{F}\left(\delta_{1}\right) x\right)=\lim _{n}{ }^{s} \hat{F}(\delta)\left({ }_{n}^{k} \hat{F}\left(\delta_{2}\right){ }_{n^{B}}^{j} \hat{F}\left(\delta_{1}\right) x\right) \\
= \pm(-1)^{\psi(s, k, j) l} \hat{F}\left(\delta \cap \delta_{2}\right){ }_{n^{B}}^{j} \hat{F}\left(\delta_{1}\right) x
\end{gathered}
$$

where $i_{s} i_{k}= \pm i_{l}, \psi(s, j, k) \in\{0,1\}$ is an integer so that $i_{s}\left(i_{j} i_{k}\right)=(-1)^{\psi(s, j, k)}\left(i_{s} i_{j}\right) i_{k}$. If a vector $x \in \bigcup_{n}{ }_{n} V_{0}$ is given, then there exists a natural number $m$ such that

$$
\text { (4) }{ }_{n}^{s} \hat{F}(\delta)\left({ }_{n^{B}}^{j} \hat{F}\left(\delta_{1}\right){ }_{n}^{k} \hat{F}\left(\delta_{2}\right) x\right)={ }^{s} \hat{F}(\delta)\left({ }_{n^{B}}^{j} \hat{F}\left(\delta_{1}\right){ }_{n}^{k} \hat{F}\left(\delta_{2}\right) x\right)
$$

for each $n>m$, consequently,

$$
\text { (5) } \quad \lim _{n}{ }_{n}^{s} \hat{F}(\delta)\left({ }_{n^{B}}^{j} \hat{F}\left(\delta_{1}\right){ }_{n}^{k} \hat{F}\left(\delta_{2}\right) x\right)={ }^{s} \hat{F}(\delta)\left({ }_{B}^{j} \hat{F}\left(\delta_{1}\right){ }^{k} \hat{F}\left(\delta_{2}\right) x\right),
$$

where $F\left(\mathcal{A}_{v}\right)=I, I$ denotes the unit operator. From Formulas $(2-5)$ and the inclusions $\bigcup_{n}{ }_{n} V=: V \subset \mathcal{D}(A) \subset \mathcal{D}(B)$ it follows, that

$$
\text { (6) }{ }^{j} \hat{B}\left({ }^{k} \hat{F}(\delta) x_{s} i_{s}\right)=(-1)^{\xi(j, k, s) k} \hat{F}(\delta)\left({ }^{j} \hat{B} x_{s} i_{s}\right)
$$

for each $x_{s} i_{s} \in V$ and $j, k, s=0,1,2, \ldots$, where $\xi(j, k, s) \in\{0,1\}$ is such integer number that $i_{j}\left(i_{k} i_{s}\right)=(-1)^{\xi(j, k, s)} i_{k}\left(i_{j} i_{s}\right)$. From the formula $i_{j}\left(i_{k} i_{s}\right)+$ $i_{k}\left(i_{j} i_{s}\right)=2 i_{s} \operatorname{Re}\left(i_{j} i_{k}\right)$ we get $(-1)^{\xi(j, k, s)}=(-1)^{\kappa(j, k)}$ for each $j, k$ and $s$, since an algebra $\operatorname{alg}_{\mathbf{R}}\left(i_{j}, i_{k}, i_{s}\right)$ over $\mathbf{R}$ generated by $i_{j}, i_{k}$ and $i_{s}$ has an embedding into the octonion algebra which is alternative [1] (see also Formulas 4.2.4(7,8) in [22]). Thus $B V \subset V$ and ${ }_{B} F V \subset V$. Then

$$
\text { (7) }{ }_{n^{H}}^{j} \hat{F}\left(\delta_{1}\right){ }_{n}^{k} \hat{F}(\delta)=(-1)^{\kappa(j, k)}{ }_{n}^{k} \hat{F}(\delta){ }_{n^{H}}^{j} \hat{F}\left(\delta_{1}\right),
$$

that is ${ }_{n} H^{*}$ quasi-permutes with ${ }_{n} F$.

In view of Lemma 2 we have ${ }_{n} H^{*}=\left.B^{*}\right|_{n V n} F\left(\mathcal{I}_{-b(n), b(n)}\right)$ and from the proof above we get

$$
\begin{gathered}
\text { (8) }{ }_{n}^{j} \hat{F}\left({ }_{B}^{k}\left(\hat{F}^{*}\right) x_{s} i_{s}\right)=(-1)^{\xi(j, k, s)}{ }_{B}^{k}\left(\hat{F}^{*}\right)\left({ }_{n}^{j} \hat{F} x_{s} i_{s}\right) \\
=(-1)^{\xi(j, k, s)}{ }_{B}^{k}\left(\hat{F}^{*}\right)\left({ }^{j} \hat{I} x_{s} i_{s}\right),
\end{gathered}
$$

consequently, $B\left({ }_{n} V\right) \subset{ }_{n} V$ and ${ }_{B} F\left({ }_{n} V\right) \subset{ }_{n} V$. Consider decomposition $x=y+z$ with $y \in{ }_{n} V$ and $z \in{ }_{n} V^{\perp}$, then $x \in \mathcal{D}(B)$ is equivalent to 
$z \in \mathcal{D}(B)$. The latter inclusion implies $z \in \mathcal{D}(B) \cap{ }_{n} V$, if additionally $x \in{ }_{n} V$, then we get $\left\langle B^{*} y ; z>=<y ; D z>=0\right.$, consequently, $B z \in$ ${ }_{n} V$ and this together with (7) leads to the inclusion ${ }_{n} F B \subset B{ }_{n} F$, that is ${ }_{n}^{j} \hat{F}^{k} \hat{B} \subset(-1)^{\kappa(j, k){ }^{k}} \hat{B}{ }_{n}^{j} \hat{F}$. For any $\mathbf{R}$-linear spaces a sign in an inclusion does not play any role. Thus ${ }_{n} V$ reduces $B$ and ${ }_{n} G$ into a normal operator ${ }_{n} Q=\left.B\right|_{n} V$.

Suppose that ${ }_{n^{G}} F$ is the canonical $\mathcal{A}_{v}$ graded projection valued measure for ${ }_{n} G$ and ${ }_{B} F$ is the canonical $\mathcal{A}_{v}$ graded projection valued measure for $B$, hence $\left.{ }_{B} F\right|_{n} V={ }_{n^{G}} F$ for each $n \in \mathbf{N}$. If $x \in \bigcup_{n}{ }_{n} V$, there exists a natural number $m$ so that

$$
\begin{gathered}
(9){ }^{j} \hat{E}\left(\delta_{1}\right)\left({ }^{k} \hat{F}\left(\delta_{2}\right) x_{s} i_{s}\right)={ }_{n^{G}}^{j} \hat{F}\left(\delta_{1}\right)\left({ }_{n}^{k} \hat{F}\left(\delta_{2}\right) x_{s} i_{s}\right) \\
=(-1)^{\xi(j, k, s)}{ }_{n}^{k} \hat{F}\left(\delta_{2}\right)\left({ }_{n}{ }^{G} \hat{F}\left(\delta_{1}\right) x_{s} i_{s}\right)
\end{gathered}
$$

for each Borel subsets $\delta_{1}$ and $\delta_{2}$ in $\mathcal{A}_{r}$, since the restriction of $A$ to ${ }_{n} V$ and ${ }_{n} G$ quasi-permute for all $n$ in accordance with Lemma 2. On the other hand, the $\mathcal{A}_{v}$ vector space $V$ is dense in $X$, consequently, ${ }_{B} F$ and $F$ quasi-permute:

$$
\text { (10) }{ }_{B}^{j} \hat{F}\left(\delta_{1}\right)^{k} \hat{F}\left(\delta_{2}\right) x_{0}=(-1)^{\kappa(j, k) k} \hat{F}\left(\delta_{2}\right){ }_{B}^{j} \hat{F}\left(\delta_{1}\right) x_{0}
$$

for each $j, k=0,1,2, \ldots$ and $x_{0} \in X_{0}$.

If now $F$ is an $\mathcal{A}_{v}$ graded projection valued measure described in this lemma, then Formula (10) implies

$$
{ }^{k} \hat{F}^{j} \hat{B} \subseteq(-1)^{\kappa(j, k) j}{ }^{j}{ }^{k} \hat{F}
$$

for each $j, k=0,1,2, \ldots, 2^{v}-1$, consequently, $\mathcal{R}(F)$ reduces $B$ and $\left.B\right|_{\mathcal{R}(F)}$ is a normal operator with $\mathcal{R}(F) \subset \mathcal{D}(B)$, since $\mathcal{R}(F) \subset \mathcal{D}(A) \subset \mathcal{D}(B)$. This restriction $\left.B\right|_{\mathcal{R}(F)}$ is bounded by the closed graph theorem 1.8.6 [12]. Moreover, the restrictions of $A$ and $B$ to $\mathcal{R}(F)$ quasi-permute. Analogous proof is valid for the pair $A$ and $C$ instead of $A$ and $B$.

5. Theorem. If operators $A, B$ and $D$ satisfy property $P$, then $B$ and D quasi-permute so that

$$
\text { (1) }{ }^{j} \hat{B}^{k} \hat{D}=(-1)^{\kappa(j, k) k} \hat{D}^{j} \hat{B}
$$


for each $j, k$. Moreover,

$$
\text { (2) }{ }^{l} \hat{A}=\sum_{j, k ; i_{j} i_{k}=i_{l}}\left({ }^{j} \hat{B}^{k} \hat{D}+(-1)^{\kappa(j, k) k} \hat{B}^{j} \hat{D}\right)
$$

for each $l$.

Proof. Consider the canonical $\mathcal{A}_{v}$ graded projection valued measure $E$ for a normal operator $A$ (see Definition 1). Then we put $\left.{ }_{n} F:=E\left(\mathcal{I}_{a, b}\right)\right)$ with $a_{j}=-n i_{j}$ and $b_{j}=n i_{j}$ for each $j$. From Theorems 2.27, 2.29 and 2.44 in [29] and $\S 4$ above we know that

$$
\begin{gathered}
\text { (3) } \quad A x=\int_{\mathcal{A}_{v}} d_{A} E(t) \cdot t x \quad \forall x \in \mathcal{D}(A) \text { and } \\
\text { (4) } \quad B x=\int_{\mathcal{A}_{r}} d_{B} E(t) \cdot t x \quad \forall x \in \mathcal{D}(B) \text { and } \\
\text { (5) } \quad D x=\int_{\mathcal{A}_{v}} d_{D} E(t) . t x \quad \forall x \in \mathcal{D}(D),
\end{gathered}
$$

where ${ }_{A} E,{ }_{B} E$ and ${ }_{D} E$ denote $\mathcal{A}_{v}$ graded projection valued measures for $A$, $B$ and $D$ respectively. Then the condition $A=B D$ gives

$$
\text { (6) } \quad A x=\int_{\mathcal{A}_{v}} d_{B} E(t) \cdot t \int_{\mathcal{A}_{v}} d_{D} E(u) \cdot u x \text {. }
$$

To operators $A, B$ and $D$ normal functions $h_{A}, h_{B}$ and $h_{D}$ correspond so that $h_{A}=h_{B} h_{D}$. On the other hand, to the operators $A^{*} A$ and $B^{*} B$ and $D^{*} D$ non-negative self-adjoint functions $\left|h_{A}\right|^{2},\left|h_{B}\right|^{2}$ and $\left|h_{D}\right|^{2}$ correspond (see Proposition 2.32 in [29]). These operators $A$ and $B$ and $D$ are normal so that they satisfy the identities $A^{*} A=D^{*} B^{*} B D=D^{*} B B^{*} D=A A^{*}=$ $B D D^{*} B^{*}=B D^{*} D B^{*}$ and $B^{*} B=B^{*} B$ and $D^{*} D=D D^{*}$.

In view of Theorems 2.29, 2.44 and Proposition 2.32 and Remark 2.43 in [29] to the $\mathcal{A}_{v}$ graded projection operator ${ }_{A} E(\delta)$ a homomorphism $\phi$ a (real) characteristic function $\phi\left({ }_{A} E(\delta)\right)=\chi_{u}$ of a subset $u \subset \Lambda$ counterpose so that $\chi_{u}=\omega\left(\chi_{\delta}\right)$. Therefore, Theorem 2.23 and Lemma 2.21 in [29], Formulas $(3-6)$ and Conditions $(P 1-P 4)$ imply that their projection operators satisfy the equality

(7) ${ }_{B} E\left(\delta_{1}\right)_{D} E\left(\delta_{2}\right)={ }_{D} E\left(\delta_{2}\right)_{B} E\left(\delta_{1}\right)$

for each Borel subsets $\delta_{1}$ and $\delta_{2}$ in $\mathcal{A}_{v}$. In view of Lemma $4 \mathcal{R}\left({ }_{n} F\right)$ reduces $B$ and $D$ and the restrictions of these operators to $\mathcal{R}\left({ }_{n} F\right)$ are bounded normal operators. On the other hand, $\bigcup_{n=1}^{\infty} \mathcal{R}\left({ }_{n} F\right)$ is dense in the Hilbert space 
$X$ over the Cayley-Dickson algebra $\mathcal{A}_{v}$. Therefore, we infer from Formulas $(3-7)$, that ${ }^{j} B$ and ${ }^{k} D$ satisfy Formulas $(1,2)$ for each $j, k$, since

$$
\text { (8) }{ }_{B}^{j} \hat{E}\left(\delta_{1}\right){ }_{D}^{k} \hat{E}\left(\delta_{2}\right)=(-1)^{\kappa(j, k)}{ }_{D}^{k} \hat{E}\left(\delta_{2}\right){ }_{B}^{j} \hat{E}\left(\delta_{1}\right)
$$

for every Borel subsets $\delta_{1}$ and $\delta_{2}$ in $\mathcal{A}_{v}$ and for each $j, k$.

6. Corollary. Suppose that operators $A, B$ and $D$ are self-adjoint and satisfy property $(P)$. Then $B D=D B$.

Proof. This follows immediately from Theorems 2.27, 2.29 and 2.44 in [29] and Formulas 5(1-3), since spectra of self-adjoint operators are contained in the real field $\mathbf{R}$ and the latter is the center of the CayleyDickson algebra $\mathcal{A}_{v}$ so that $t=t_{0} \in \mathbf{R}$ in Formulas $5(1,2)$, that is $j=k=0$ only.

7. Lemma. Let operators $B, D$ and $A$ have property $P$, let also $B=$ $T_{B} U_{B}, \quad D=T_{D} U_{D}$ and $A=T U$ be their canonical decompositions with positive self-adjoint operators $T_{B}, T_{D}$ and $T$ and unitary operators $U_{B}, U_{D}$ and $U$ respectively. Then $T_{B} T_{D}=T_{D} T_{B}=T$ and $U_{B} U_{D}=U$ so that ${ }^{j} U_{B}{ }^{k} U_{D}=(-1)^{\kappa(j, k)}{ }^{j} U_{B}{ }^{k} U_{D}$ for each $j, k$, moreover, $T_{B} U_{D}=U_{D} T_{B}$ and $T_{D} U_{B}=U_{B} T_{D}$.

Proof. The decompositions in the conditions of this lemma are particular cases of that of Theorem I.3.37 [28]. Consider the canonical $\mathcal{A}_{v}$ graded resolutions of the identity $E^{B}$ and $E^{D}$ of operators $B$ and $D$ respectively. In view of Theorem 5

$$
{ }^{j} E^{B}\left(\delta_{1}\right)^{k} E^{D}\left(\delta_{2}\right)=(-1)^{\kappa(j, k) k} E^{D}\left(\delta_{2}\right)^{j} E^{B}\left(\delta_{1}\right)
$$

for every Borel subsets $\delta_{1}$ and $\delta_{2}$ in $\mathcal{A}_{r}$ and each $j, k$. We put $F(d w, d z)=$ $E^{B}(d w) E^{D}(d z)$, hence $F(d w, d z)$ is a $2^{v+1}$ parameter $\mathcal{A}_{v}$ graded resolution of the identity so that $F_{i_{k}}\left(\delta_{1}, \delta_{2}\right) x_{k}=E^{B}\left(\delta_{1}\right)\left(E^{D}\right)_{i_{k}}\left(\delta_{2}\right)$ for each vector $x_{k} \in X_{k}$ and every $k$ and we put

$$
G:=\int_{\mathcal{A}_{v}^{2}} d F(w, z) \cdot w z
$$

where $d F(w, z)$ is another notation of $F(d w, d z), w, z \in \mathcal{A}_{v}$ (see also $\S I .2 .58$ [28]). This operator $G$ is normal, since the quaternion skew field is associative 
and the octonion algebra is alternative and $(w z)(w z)^{*}=|w z|^{2}=|w|^{2}|z|^{2}$ for each $w, z \in \mathcal{A}_{v}$ with $2 \leq v \leq 3$. Then we get

$$
\begin{gathered}
B=\int_{\mathcal{A}_{v}^{2}} d F(w, z) \cdot w=\int_{\mathcal{A}_{v}} d E^{B}(w) \cdot w \text { and } \\
D=\int_{\mathcal{A}_{v}^{2}} d F(w, z) \cdot z=\int_{\mathcal{A}_{v}} d E^{D}(z) \cdot z, \text { consequently, }
\end{gathered}
$$

$A=B D$ and ${ }^{j} B{ }^{k} D=(-1)^{\kappa(j, k) k} D^{j} B$ for each $j, k$, and hence

$$
\sum_{j, k: i_{j} i_{k}=i_{l}}\left[{ }^{j} B{ }^{k} D+(-1)^{\kappa(j, k) k} B{ }^{j} D\right] \subseteq{ }^{l} G
$$

for every $l$. Therefore, $A=G$, since a normal operator is maximal.

Then one can consider the function $u(w, z):=w z /|w z|$ for $w z \neq 0$, while $u(w, z)=1$ if $w z=0$, where $w, z \in \mathcal{A}_{v}$. The operator

$$
U:=\int_{\mathcal{A}_{v}^{2}} d F(w, z) \cdot u(w, z)
$$

is unitary, since $|u(w, z)|=1$ for each $w$ and $z$, the operator

$$
T:=\int_{\mathcal{A}_{v}^{2}} d F(w, z) \cdot|w z|
$$

is positive and self-adjoint, since

$$
<x T ; x>:=\int_{\mathcal{A}_{v}^{2}}<x d F(w, z) .|w z| ; x>\geq 0
$$

for each $x \in \mathcal{D}(T)$ (see Proposition 2.35 [29]). On the other hand, $u(w, z)|w z|=$ $|w z| u(w, z)=w z$, since the algebra $\mathcal{A}_{v}$ is alternative for $v \leq 3$, hence $T U=U T=G=A$ by Theorem 2.44 [29]. Moreover, we deduce from Theorem 2.44 [29] that the operators

$$
\begin{gathered}
U_{B}:=\int_{\mathcal{A}_{v}^{2}} d F(w, z) \cdot u(w) \text { and } \\
U_{D}:=\int_{\mathcal{A}_{v}^{2}} d F(w, z) \cdot u(z)
\end{gathered}
$$

are unitary and the the operators

$$
\begin{gathered}
T_{B}:=\int_{\mathcal{A}_{v}^{2}} d F(w, z) \cdot|w| \text { and } \\
T_{D}:=\int_{\mathcal{A}_{r}^{2}} d F(w, z) \cdot|z|
\end{gathered}
$$


are positive and self-adjoint, where $u(w):=w /|w|$ if $w \neq 0$, also $u(w)=1$ if $w=0$. Since $|w||z|=|w z|$ for each $w$ and $z \in \mathcal{A}_{v}$ with $v \leq 3$, the inclusion follows

$$
T_{B} T_{D} \subseteq \int_{\mathcal{A}_{v}^{2}} d F(w, z) \cdot|w z|=T .
$$

The functions $u(w)$ and $u(z)$ are bounded and $u(w) u(z)=u(z) u(w)=$ $u(w, z)$ on $\mathcal{A}_{v}^{2}$, consequently,

$$
U_{B} U_{D}=\int_{\mathcal{A}_{v}^{2}} d F(w, z) \cdot u(w, z)=U \text { so that }
$$

${ }^{j} U_{B}{ }^{k} U_{D}=(-1)^{\kappa(j, k) k} U_{B}{ }^{j} U_{D}$ for each $j, k$. This implies that $A=U T=$ $U_{B} U_{D} T=\left(U_{B} T_{B}\right)\left(U_{D} T_{D}\right)=\left(U_{B} T_{B}\right)\left(T_{D} U_{D}\right)$, consequently, $U_{D} T U_{D}^{*}=T_{B} T_{D}$. This means that the operators $T$ and $T_{B} T_{D}$ are unitarily equivalent, hence the operator product $T_{B} T_{D}$ is self-adjoint. A self-adjoint operator is maximal, consequently, $T=T_{B} T_{D}$ and similarly $T=T_{D} T_{B}$. The real field $\mathbf{R}$ is the center of the Cayley-Dickson algebra $\mathcal{A}_{v}$ for each $v \geq 2$, the real and complex fields are commutative, hence

$$
\begin{gathered}
T_{B} U_{D}=\int_{\mathcal{A}_{v}^{2}} d F(w, z) \cdot(|w| u(z))=U_{D} T_{B} \text { and } \\
T_{D} U_{B}=\int_{\mathcal{A}_{v}^{2}} d F(w, z) \cdot(|z| u(w))=U_{B} T_{D} .
\end{gathered}
$$

8. Notation. Let $\Omega$ denote the set of all $n$-tuples $x=\left(x_{1}, \ldots, x_{m}, x_{m+1}, \ldots, x_{n}\right)$ such that $x_{1}, \ldots, x_{m}$ are non-negative integers, while $x_{m+1}, \ldots, x_{n}$ are nonnegative real numbers with $\sum_{j=1}^{n} x_{j}>0$. Relative to the addition $x+y=$ $\left(x_{1}+y_{1}, \ldots, x_{n}+y_{n}\right)$ this set $\Omega$ forms a semi-group.

9. Theorem. Suppose that $\left\{B^{x}: x \in \Omega\right\}$ is a weakly continuous semigroup of normal operators, that is satisfying the following conditions:

(1) $B^{x}$ is a normal operator acting on a Hilbert space $X$ over the CayleyDickson algebra $\mathcal{A}_{v}$ for each element $x \in \Omega$;

(2) $B^{x} B^{y}=B^{x+y}$ for each $x, y \in \Omega$;

(3) the $\mathcal{A}_{v}$ valued scalar product $\left\langle B^{x} f ; g>\right.$ is continuous in $x \in \Omega$ for each marked $f, g \in \mathcal{D}:=\bigcap_{x \in \Omega} \mathcal{D}\left(B^{x}\right)$;

(4) a family alg $\mathcal{A}_{v}\left\{I, B^{x},\left(B^{x}\right)^{*}: x \in \Omega\right\}$ is over the algebra $\mathcal{A}_{v}$ with $2 \leq$ $v \leq 3$. Then a unique $2 n$-parameter $\mathcal{A}_{v}$ graded resolution $\left\{\left(a_{1}, \ldots, a_{n} ; b_{1}, \ldots, b_{n}\right) \hat{F}: a, b \in\right.$ 
$\Omega\}$ of the identity exists so that ${ }_{(a, b)} \hat{F}=0$ if a negative coordinate $a_{k}<0$ exists for some $k=1, \ldots, n$, moreover,

(5) $\quad B^{x}=\int_{\mathbf{R}^{2 n}} d{ }_{(a, b)} \hat{F} \cdot\left\{a^{x} \exp \left[x_{1} M_{1}\left(b_{1}\right) b_{1}\right] \ldots \exp \left[x_{n} M_{n}\left(b_{n}\right) b_{n}\right]\right\}$,

where

$$
a^{x}=\prod_{k=1}^{n} a_{k}^{x_{k}}
$$

$M_{s}: \mathbf{R}^{n} \rightarrow \mathcal{S}_{v}:=\left\{z \in \mathcal{A}_{v}:|z|=1, \operatorname{Re}(z)=0\right\}$ is a Borel function for each $s, \quad a=\left(a_{1}, \ldots, a_{n}\right)$.

Proof. In view of Lemma 5 each operator $B^{x}$ has the decomposition $B^{x}=T^{x} U^{x}=U^{x} T^{x}$ with a positive self-adjoint operator $T^{x}$ and a unitary operator $U^{x}$. Since $\left\{B^{x}: x \in \Omega\right\}$ is a semi-group, the relations $T^{x} T^{y}=T^{x+y}$ and $U^{x} U^{y}=U^{x+y}$ are valid for each elements $x, y \in \Omega$. That is, $\left\{T^{x}: x \in \Omega\right\}$ and $\left\{U^{x}: \quad x \in \Omega\right\}$ are semi-groups of positive self-adjoint operators and unitary operators correspondingly.

If $y^{s}=\left(0, \ldots, y_{m+1}^{s}, \ldots, y_{n}^{s}\right) \in \Omega$ are elements of the semi-group $\Omega$ such that $y^{1}=\frac{y^{2}+y^{3}}{2}, s=1,2,3, f$ is a vector in a domain $\mathcal{D}$, then

$$
\begin{gathered}
\left\|B^{y^{1}} f\right\|^{2}=<B^{y^{1}} f, B^{y^{1}} f>=<B^{y^{2} / 2} B^{y^{3} / 2} f ; B^{y^{2} / 2} B^{y^{3} / 2} f> \\
=<\left(B^{y^{2} / 2}\right)^{*} B^{y^{2} / 2} f ;\left(B^{y^{3} / 2}\right)^{*} B^{y^{3} / 2} f>\leq\left\|\left(B^{y^{2} / 2}\right)^{*} B^{y^{2} / 2} f\right\|\left\|\left(B^{y^{3} / 2}\right)^{*} B^{y^{3} / 2} f\right\|
\end{gathered}
$$

by Cauchy-Schwartz' inequality I.2.4(1) [28]. On the other hand, $\left\|\left(B^{y^{2} / 2}\right)^{*} B^{y^{2} / 2} f\right\|^{2}=<\left(B^{y^{2} / 2}\right)^{*} B^{y^{2} / 2} f ;\left(B^{y^{2} / 2}\right)^{*} B^{y^{2} / 2} f>=<B^{y^{2}} f ; B^{y^{2}} f>=\left\|B^{y^{2}} f\right\|^{2}$, since the semi-group $\left\{B^{x}: x \in \Omega\right\}$ is commutative and an operator $B^{x}$ is normal for each $x \in \Omega$. Thus the inequality

$$
\left\|B^{y^{1}} f\right\| \leq\left\|B^{y^{2}} f\right\|\left\|B^{y^{3}} f\right\|
$$

follows. This implies that the function $q(y):=\left\|B^{y} f\right\|$ is convex and bounded in the variable $y_{p}$ in any bounded segment $[\alpha, \beta] \subset(0, \infty)$, when other variables $y_{q}$ with $q \neq p$ are zero, $p=m+1, \ldots, n$, since the exponential $e^{t}$ and the natural logarithmic functions $\ln (t)$ are convex and bounded on each segment $[\gamma, \delta] \subset(0, \infty)$ and $\ln q\left(y^{1}\right) \leq \ln q\left(y^{2}\right)+\ln q\left(y^{3}\right)$.

Evidently, a commutative group $\hat{\Omega}$ exists for the semi-group $\Omega$ such that $\Omega \subset \hat{\Omega} \subset \mathbf{R}^{n}$ and the function $q(y)$ can be extended on $\hat{\Omega}$ so that $q(0)=\|f\|$ 
and $q(-y)=q(y)$ for $y \in \Omega$. If $q$ is continuous on $\Omega$, its extension on $\hat{\Omega}$ can be chosen continuous, since $\hat{\Omega}$ is a completely regular topological space, i.e. $T_{1}$ and $T_{3.5}$ (see [6]).

If $\Omega$ is a group the function $q(y)$ is positive definite, that is by the definition for each $\lambda_{1}, \ldots, \lambda_{k} \in \mathbf{R} \oplus \mathbf{R i}=: \mathbf{C}_{\mathbf{i}}$ and $y^{1}, \ldots, y^{k} \in \Omega$ the inequality

$$
\sum_{j, l} \lambda_{j} \bar{\lambda}_{l} q\left(y^{j}-y^{l}\right) \geq 0
$$

is valid, but this inequality follows from the formula

$$
\sum_{j, l} \lambda_{j} \bar{\lambda}_{l} q\left(y^{j}-y^{l}\right)=\left\|\sum_{j} \lambda_{j} B^{y^{j}} f\right\|^{2}
$$

and since $\|x\| \geq 0$ for each $x \in X$.

Particularly, for elements $x^{k}:=\left(0, \ldots, x_{k}, 0, \ldots, 0\right)$ in the semi-group $\Omega$ the mapping $<T^{x^{k}} f ; f>$ is continuous in $x^{k}$ for each marked vector $f \in \mathcal{D}$. Indeed, for $k=1, \ldots, m$ this is evident, since $x^{k} \in \mathbf{N}$ takes values in the discrete space in this case. If $k=m+1, \ldots, n$ one can use the formula $<$ $T^{x^{k}} f ; f>=<B^{x^{k} / 2} f, B^{x^{k} / 2} f>=\left\|B^{x^{k} / 2} f\right\|^{2}$ which implies that $<T^{x^{k}} f ; f>$ is a bounded convex function of $x^{k}$ in every finite interval $[\alpha, \beta] \subset(0, \infty)$, when $f \in \mathcal{D}$ is a marked vector (see Theorem 2.29 and Formula 2.44(5) [29]).

Denote by ${ }_{s, t_{s}} E$ the canonical $\mathcal{A}_{v}$ graded resolution of the identity for $T^{e_{s}}$, where $e_{s}=(0, \ldots, 0,1,0, \ldots)$ denotes the basic vector with coordinate 1 at $s$-th place and zeros otherwise, $t_{s} \in \mathbf{R}$. By the conditions of this theorem operators $T^{e_{s}}$ and $T^{e_{p}}$ commute for each $s, p=1, \ldots, n$, since

(6) $T^{e_{s}} T^{e_{p}}=T^{e_{s}+e_{p}}=T^{e_{p}} T^{e_{s}}$.

Due to Theorem 2.42 [29] the equality

$$
\text { (7) }{ }_{s, t_{s}}^{j} E_{p, t_{p}}^{k} E=(-1)^{\kappa(j, k)} \underset{p, t_{p}}{k} E_{s, t_{s}}^{j} E
$$

is satisfied for each $j, k$ and every $s, p$, with $t_{s}, t_{p} \in \mathbf{R}$. This implies that

(8) ${ }_{\left(t_{1}, \ldots, t_{n}\right)} E={ }_{1, t_{1}} E \ldots n, t_{n} E$

is an $n$-parameter $\mathcal{A}_{v}$ graded resolution of the identity. Each operator $T^{e_{s}}$ is positive, hence ${ }_{s, t_{s}} E=0$ for every $t_{s}<0$, consequently, ${\left(t_{1}, \ldots, t_{n}\right)} E=0$ if $t_{s}<0$ for some $s=1, \ldots, n$.

We now consider the operators

$$
\text { (9) } \quad A^{p} x:=\int_{0}^{\infty} \cdots \int_{0}^{\infty} d_{\left(t_{1}, \ldots, t_{n}\right)} E \cdot\left(t_{1}^{p_{1}} \ldots t_{n}^{p_{n}} x\right) \text {, }
$$


where $p=\left(p_{1}, \ldots, p_{n}\right) \in \Omega, \quad x \in X$ for which the integral converges. We certainly have

$$
\int_{0}^{\infty} \ldots \int_{0}^{\infty} d_{\left(t_{1}, \ldots, t_{n}\right)} E .\left(t_{1}^{p_{1}} \ldots t_{n}^{p_{n}} x\right)=\int_{0}^{\infty} \ldots \int_{0}^{\infty}\left(t_{1}^{p_{1}} \ldots t_{n}^{p_{n}}\right) d_{\left(t_{1}, \ldots, t_{n}\right)} E . x,
$$

since $t_{j}^{p_{j}} \in \mathbf{R}$ for each $j$ and ${ }_{\left(t_{1}, \ldots, t_{n}\right)} E$ is a real linear operator. If $p_{s} \in \mathbf{Z} / 2$ for each $s$, then $T^{p}=T^{e_{1} p_{1}} \ldots T^{e_{n} p_{n}} \subseteq A^{p}$, consequently, $T^{p}=A^{p}$, since a self-adjoint operator is maximal.

Take a partition of the Euclidean space $\mathbf{R}^{n}$ into a countable family of bounded parallelepipeds $J_{k}=\prod_{j=1}^{n}\left[a_{j}, b_{j}\right]$ so that they may intersect only by their boundaries: $J_{k} \cap J_{l}=\partial J_{k} \cap \partial J_{l}$ for each $k \neq l \in \mathbf{N}, \quad \bigcup_{k=1}^{\infty} J_{k}=\mathbf{R}^{n}$. We put $Y^{k}:=\mathcal{R}\left(\hat{\mathbf{E}}\left(J_{k}\right)\right)$, where $\hat{\mathbf{E}}(\delta)$ is the $\mathcal{A}_{v}$ graded spectral measure corresponding to ${ }_{t} E, \quad \delta \in \mathcal{B}\left(\mathbf{R}^{n}\right), \quad t \in \mathbf{R}^{n}$. Then the restriction $\left.B^{x}\right|_{Y^{k}}$ of $B^{x}$ to $Y^{k}$ is a bounded self-adjoint operator. If $x, y \in \Omega$ are elements of the semi-group so that $y_{s} \geq x_{s}$ and $y_{s} \in \mathbf{Z} / 2$ for each $s=1, \ldots, n$, then $\mathcal{D}\left(T^{y}\right) \subseteq$ $\mathcal{D}\left(T^{x}\right)$, since $T^{y}=T^{x} T^{y-x}$. Therefore, $f \in \mathcal{D}\left(A^{y}\right)=\mathcal{D}\left(T^{y}\right) \subseteq \mathcal{D}\left(T^{x}\right)$ for each $f \in Y^{k}$, consequently, $Y^{k} \subset \mathcal{D}$ for each natural number $k \in \mathbf{N}$.

If $f \in Y^{k} \oplus Y^{l}$ and $g \in Y^{l}$, then

$$
\lim _{y \rightarrow x}<\left(T^{y}-A^{y}\right)(f+g) ;(f+g)>=<\left(T^{x}-A^{x}\right)(f+g) ;(f+g)>=0,
$$

since $T^{y}=A^{y}$ for each $y \in(\mathbf{Z} / 2)^{n} \cap \Omega$ and the $\mathcal{A}_{v}$ valued scalar products $<T^{x} f ; f>$ and $<A^{x} f ; f>$ are continuous in each component $x_{s}$ of $x$. In the same manner we get $\left\langle\left(T^{x}-A^{x}\right) f ; f>=0\right.$ and $\left\langle\left(T^{x}-A^{x}\right) g ; g>=0\right.$, consequently, $<\left(T^{x}-A^{x}\right) f ; g>=0$. The $\mathcal{A}_{v}$ vector space $\bigcup_{k=1}^{\infty} Y^{k}$ is dense in the Hilbert space $X$ over the Cayley-Dickson algebra $\mathcal{A}_{v}$, hence $T^{x} f^{k}=$ $\left(\left.A^{x}\right|_{Y^{k}}\right) f^{k}=A^{x} f^{k}$ for each vector $f^{k} \in Y^{k}$. This means that each $Y^{k}$ reduces the operator $T^{x}$ to $\left(\left.A^{x}\right|_{Y^{k}}\right)$, consequently, $T^{x}=A^{x}$. From this it follows that the $\mathcal{A}_{v}$ valued scalar product $\left\langle T^{x} f ; g\right\rangle$ is continuous in $x \in \Omega$ for each marked vectors $f \in \mathcal{D}$ and $g \in X$.

Consider the sub-semi-group $\Omega_{s}:=\left\{x: x=x^{s}:=\left(0, \ldots, 0, x_{s}, 0, \ldots\right) \in\right.$ $\Omega\}$, where $s=1, \ldots, n$, also we suppose that ${ }_{s} \hat{\mathbf{E}}(\{0\})=0$, where ${ }_{s} \hat{\mathbf{E}}(\delta)$ is the $\mathcal{A}_{v}$ graded projection valued measure corresponding to ${ }_{s, t_{s}} E, \delta \in \mathcal{B}(\mathbf{R})$. This implies that the operator $T^{x^{s}}$ has not the zero eigenvalue. Take arbitrary marked vectors $f \in \mathcal{D}$ and $g \in \mathcal{D}\left(T^{y^{s}}\right)$. Then using the triangle inequality 
we deduce that

$$
\begin{aligned}
& \left|<\left(U^{x^{s}}-U^{y^{s}}\right) f ; T^{y^{s}} g>\right|=\left|<\left(U^{x^{s}}-U^{y^{s}}\right) T^{y^{s}} f ; g>\right|=\mid<\left(U^{x^{s}} T^{x^{s}}-U^{y^{s}} T^{y^{s}}\right) f ; g> \\
& +<U^{x^{s}}\left(T^{y^{s}}-T^{x^{s}}\right) f ; g>|\leq|<\left(B^{x^{s}}-B^{y^{s}}\right) f ; g>\mid+\left\|\left(T^{y^{s}}-T^{x^{s}}\right) f\right\|\|g\| .
\end{aligned}
$$

But the limits are zero $\lim _{x^{s} \rightarrow y^{s}}<\left(B^{x^{s}}-B^{y^{s}}\right) f ; g>=0$ due to suppositions of this theorem and $\lim _{x^{s} \rightarrow y^{s}}\left\|\left(T^{y^{s}}-T^{x^{s}}\right) f\right\|=0$, since $T^{x}=A^{x}$ and $A^{x}$ has the integral representation given by Formula (9). Thus the limit

$$
\lim _{x^{s} \rightarrow y^{s}}<\left(U^{x^{s}}-U^{y^{s}}\right) f ; h>=0
$$

is zero for each $f \in \mathcal{D}$ and $h \in \mathcal{R}\left(T^{y^{s}}\right)$. On the other hand, $\mathcal{D}$ is dense in $X$, since $\bigoplus_{k=1}^{\infty} Y^{s}$ is dense in $X$. The family $U^{x}$ of unitary operators is norm bounded by the unit 1 , consequently, $\lim _{x^{s} \rightarrow y^{s}}<\left(U^{x^{s}}-U^{y^{s}}\right) f ; h>=0$ for each $f, h \in X$ and hence the semi-group $\left\{U^{x^{s}}: x^{s} \in \Omega\right\}$ is weakly continuous. The semi-group $\left\{U^{x^{s}}: x^{s} \in \Omega\right\}$ of unitary operators can be extended to a weakly continuous group of unitary operators putting $U^{-x^{s}}=\left(U^{x^{s}}\right)^{*}$ and $U^{0}=I$. This one-parameter commutative group of unitary operators is also strongly continuous, since

$$
\begin{gathered}
\left\|\left(U^{x^{s}}-U^{y^{s}}\right) f\right\|^{2}=<\left(U^{x^{s}}-U^{y^{s}}\right) f ;\left(U^{x^{s}}-U^{y^{s}}\right) f>= \\
<\left(U^{x^{s}}-U^{y^{s}}\right)^{*}\left(U^{x^{s}}-U^{y^{s}}\right) f ; f>=<\left(2 I-U^{x^{s}-y^{s}}-U^{y^{s}-x^{s}}\right) f ; f> \\
=<\left(U^{0}-U^{x^{s}-y^{s}}\right) f ; f>+<\left(U^{0}-U^{y^{s}-x^{s}}\right) f ; f>.
\end{gathered}
$$

In view of Theorem I.3.28 [28] there exists a unique $\mathcal{A}_{v}$ graded projection valued measure ${ }_{s} \hat{\mathbf{F}}$ so that

$$
\text { (10) }<U\left(x^{s}\right) f ; h>=\int_{-\infty}^{\infty}<{ }_{s} \hat{\mathbf{F}}\left(d b_{s}\right) \cdot \exp \left(x_{s} M_{s}\left(b_{s}\right) b_{s}\right) f ; h>
$$

for each $f, h \in \mathcal{D}\left(Q^{s}\right)$, where

$$
\text { (11) }<Q^{s} f, h>=\int_{-\infty}^{\infty} b_{s}<{ }_{s} \hat{\mathbf{F}}\left(d b_{s}\right) f ; h>
$$

for each $f, h \in \mathcal{D}\left(Q^{s}\right)$,

$$
\mathcal{D}\left(Q^{s}\right)=\left\{f: f \in X ;\left\|Q^{s} f\right\|^{2}=\int_{-\infty}^{\infty}<{ }_{s} \hat{\mathbf{F}}\left(d b_{s}\right) . b_{s}^{2} f ; f><\infty\right\}
$$


$M_{s}\left(b_{s}\right)$ is a Borel function from $\mathbf{R}$ into the purely imaginary unit sphere $\mathcal{S}_{v}:=$ $\left\{z \in \mathcal{A}_{v}:|z|=1, \operatorname{Re}(z)=0\right\}$. Then we put ${ }_{s} \hat{\mathbf{E}}\left(d a_{s}, d b_{s}\right)={ }_{s} \hat{\mathbf{E}}\left(d a_{s}\right){ }_{s} \hat{\mathbf{F}}\left(d b_{s}\right)$, where

$$
{ }_{s}^{j} \hat{\mathbf{E}}\left(\delta_{1}\right){ }_{s}^{k} \hat{\mathbf{F}}\left(\delta_{2}\right)=(-1)^{\kappa(j, k)}{ }_{s}^{k} \hat{\mathbf{E}}\left(\delta_{1}\right){ }_{s}^{j} \hat{\mathbf{F}}\left(\delta_{2}\right)
$$

for each $j, k$ and Borel subsets $\delta_{1}, \delta_{2} \in \mathcal{B}(\mathbf{R})$. Then an operator $P^{x^{s}}$ exists prescribed by the formula:

$$
P^{x^{s}}=\int_{-\infty}^{\infty} \int_{0}^{\infty} \hat{\mathbf{E}}\left(d a_{s}, d b_{s}\right) \cdot\left[a_{s}^{x_{s}} \exp \left(x_{s} M_{s}\left(b_{s}\right) b_{s}\right)\right]
$$

This implies the inclusion $B^{x^{s}} \subseteq P^{x^{s}}$, but a normal operator is maximal, consequently, $B^{x^{s}}=P^{x^{s}}$ for each $s$ and $x^{s} \in \Omega$.

Suppose now that ${ }_{s} \hat{\mathbf{E}}(\{0\}) \neq 0$, consider the null space $N^{s}:=\operatorname{ker}\left(B^{x^{s}}\right)$ of $B^{x^{s}}$. To each $\mathcal{A}_{v}$ graded projection valued measure ${ }_{s} \hat{\mathbf{E}}(\delta)$ associated with the family $\operatorname{alg}_{\mathcal{A}_{v}}\left(I, B^{x},\left(B^{x}\right)^{*}\right)$ a real valued characteristic function in $\mathcal{N}(\Lambda, \mathbf{R})$ corresponds, where $\delta \in \mathcal{B}\left(\mathbf{R}^{2}\right)$, consequently, $N^{s}$ is an $\mathcal{A}_{v}$ vector subspace in $X$. Let $X=N^{s} \oplus K^{s}$, hence $K^{s}$ is an $\mathcal{A}_{v}$ vector space, since $N^{s}$ is the $\mathcal{A}_{v}$ vector subspace of the $\mathcal{A}_{v}$ Hilbert space $X$. Take the restrictions $\left.B^{x^{s}}\right|_{N^{s}}=: B^{x^{s}, N}$ and $\left.B^{x^{s}}\right|_{K^{s}}=: B^{x^{s}, K}$ of $B^{x^{s}}$ to $N^{s}$ and $K^{s}$ correspondingly. This implies that the semi-group of normal operators $\left\{B^{x^{s}, K}: x^{s} \in \Omega\right\}$ possesses the property that none of the operators $B^{x^{s}, K}$ has zero eigenvalue. From Formula (13) it follows, that there exists a two-parameter resolution ${ }_{s, K} \hat{\mathbf{E}}$ of the identity so that

$$
\text { (14) } \quad B^{x^{s}, K}=\int_{-\infty}^{\infty} \int_{0}^{\infty}{ }_{s, K} \hat{\mathbf{E}}\left(d a_{s}, d b_{s}\right) \cdot\left[a_{s}^{x_{s}} \exp \left(x_{s} M_{s}\left(b_{s}\right) b_{s}\right)\right]
$$

Define an $\mathcal{A}_{v}$ graded projection value measure ${ }_{s, N} \hat{\mathbf{E}}$ so that

$$
\int_{-\infty}^{a_{s}} \int_{-\infty}^{b_{s}}{ }_{s, N} \hat{\mathbf{E}}\left(d t_{s}, d q_{s}\right)={ }_{s, N ; a_{s}, b_{s}} \hat{\mathbf{E}}
$$

so that ${ }_{s, N ; a_{s}, b_{s}} \hat{\mathbf{E}}=0$ for $a_{s}<0$ and ${ }_{s, N ; a_{s}, b_{s}} \hat{\mathbf{E}}=I$ when $a_{s} \geq 0$. Since $B^{x^{s}, N}\left(N^{s}\right)=\{0\}$, the integral representation follows:

$$
\text { (15) } \quad B^{x^{s}, N}=\int_{-\infty}^{\infty} \int_{0}^{\infty}{ }_{s, N} \hat{\mathbf{E}}\left(d a_{s}, d b_{s}\right) \cdot\left[a_{s}^{x_{s}} \exp \left(x_{s} M_{s}\left(b_{s}\right) b_{s}\right)\right]
$$

Now it is natural to put ${ }_{s} \hat{\mathbf{E}}\left(d a_{s}, d b_{s}\right)={ }_{s, N} \hat{\mathbf{E}}\left(d a_{s}, d b_{s}\right) \oplus_{s, K} \hat{\mathbf{E}}\left(d a_{s}, d b_{s}\right)$ for an $\mathcal{A}_{v}$ graded projection valued measure on $X$, that induces the formula:

$$
B^{x^{s}}=\int_{-\infty}^{\infty} \int_{0}^{\infty}{ }_{s} \hat{\mathbf{E}}\left(d a_{s}, d b_{s}\right) \cdot\left[a_{s}^{x_{s}} \exp \left(x_{s} M_{s}\left(b_{s}\right) b_{s}\right)\right]
$$


In accordance with Theorem 5

$$
{ }_{s}^{j} \hat{\mathbf{E}}\left(\delta_{1}\right){ }_{q}^{k} \hat{\mathbf{E}}\left(\delta_{2}\right)=(-1)^{\kappa(j, k)}{ }_{q}^{k} \hat{\mathbf{E}}\left(\delta_{2}\right){ }_{s}^{j} \hat{\mathbf{E}}\left(\delta_{1}\right)
$$

for each $s, q=1, \ldots, n$ and $j, k=0,1, \ldots, 2^{v}-1$ and every $\delta_{1}, \delta_{2} \in \mathcal{B}\left(\mathbf{R}^{2}\right)$, particularly, for $j=k=0$ i.e. ${ }_{s} \hat{\mathbf{E}}\left(\delta_{1}\right)$ and ${ }_{q} \hat{\mathbf{E}}\left(\delta_{2}\right)$ commute. Then we put

$$
{ }_{(a, b)} \hat{F}=\int_{-\infty}^{a_{1}} \int_{-\infty}^{b_{1}} \ldots \int_{-\infty}^{a_{n}} \int_{-\infty}^{b_{n}}{ }_{1} \hat{\mathbf{E}}\left(d t_{1}, d q_{1}\right) \cdots_{n} \hat{\mathbf{E}}\left(d t_{n}, d q_{n}\right),
$$

hence ${ }_{(a, b)} \hat{F}$ is an $\mathcal{A}_{v}$ graded resolution of the identity, for which

$$
\begin{gathered}
d_{(a, b)} \hat{F} \cdot\left\{a^{x} \exp \left[x_{1} M_{1}\left(b_{1}\right) b_{1}\right] \ldots \exp \left[x_{n} M_{n}\left(b_{n}\right) b_{n}\right]\right\}= \\
{ }_{1} \hat{\mathbf{E}}\left(d a_{1}, d b_{1}\right) \cdot \exp \left(x_{1} M_{1}\left(b_{1}\right) b_{1}\right) \ldots_{n} \hat{\mathbf{E}}\left(d a_{n}, d b_{n}\right) \cdot \exp \left(x_{n} M_{n}\left(b_{n}\right) b_{n}\right) a^{x},
\end{gathered}
$$

since the semi-groups $\left\{B^{x}: x \in \Omega\right\}$ and $\left\{T^{x}: x \in \Omega\right\}$ and $\left\{U^{x}: x \in \Omega\right\}$ are commutative, the real field $\mathbf{R}$ is the center of the Cayley-Dickson algebra $\mathcal{A}_{v}$ for each $v \geq 2$, the fields $\mathcal{A}_{0}=\mathbf{R}$ and $\mathcal{A}_{1}=\mathbf{C}$ are commutative, $a_{s} \in \mathbf{R}$ and $x_{s} \in \mathbf{R}$ for each $s=1, \ldots, n$. For the operators

$$
P^{x}=\int_{\mathbf{R}^{2 n}} d_{(a, b)} \hat{F} \cdot\left\{a^{x} \exp \left[x_{1} M_{1}\left(b_{1}\right) b_{1}\right] \ldots \exp \left[x_{n} M_{n}\left(b_{n}\right) b_{n}\right]\right\}
$$

where

$$
a^{x}=\prod_{k=1}^{n} a_{k}^{x_{k}}
$$

$M_{s}: \mathbf{R}^{n} \rightarrow \mathcal{S}_{v}:=\left\{z \in \mathcal{A}_{v}:|z|=1, \operatorname{Re}(z)=0\right\}$ is a Borel function for each $s$, the inclusion follows $B^{x} \subseteq P^{x}$ for each $x \in \Omega$, since $B^{x}=B^{x^{1}} \ldots B^{x^{n}}$, where the operators $B^{x^{1}}, \ldots, B^{x^{n}}$ pairwise commute. But a normal operator is maximal, consequently, $B^{x}=P^{x}$ for each $x \in \Omega$. A uniqueness of the resolution ${ }_{(a, b)} \hat{F}$ of the identity follows from uniqueness of ${ }_{s} \hat{\mathbf{F}}$ and ${ }_{s} \hat{\mathbf{E}}$ for each $s$.

\section{References}

[1] J.C. Baez. "The octonions". Bull. Amer. Mathem. Soc. 39: 2 (2002), 145-205.

[2] F. Brackx, R. Delanghe, F. Sommen. "Clifford analysis" (London: Pitman, 1982). 
[3] L.E. Dickson. "The collected mathematical papers". Volumes 1-5 (Chelsea Publishing Co.: New York, 1975).

[4] N. Dunford, J.C. Schwartz. "Linear operators" (J. Wiley and Sons, Inc.: New York, 1966).

[5] G. Emch. "Mèchanique quantique quaternionienne et Relativitè restreinte". Helv. Phys. Acta 36 (1963), 739-788.

[6] R. Engelking. "General topology" (Heldermann: Berlin, 1989).

[7] J.E. Gilbert, M.A.M. Murray. "Clifford algebras and Dirac operators in harmonic analysis". Cambr. studies in advanced Mathem. 26 (Cambr. Univ. Press: Cambridge, 1991).

[8] P.R. Girard. "Quaternions, Clifford algebras and relativistic Physics" (Birkhäuser: Basel, 2007).

[9] K. Gürlebeck, W. Sprössig. "Quaternionic analysis and elliptic boundary value problem" (Birkhäuser: Basel, 1990).

[10] F. Gürsey, C.-H. Tze. "On the role of division, Jordan and related algebras in particle physics" (World Scientific Publ. Co.: Singapore, 1996).

[11] M. Junge, Q. Xu. "Representation of certain homogeneous Hilbertian operator spaces and applications". Invent. Mathematicae 179: 1 (2010), 75-118.

[12] R.V. Kadison, J.R. Ringrose. "Fundamentals of the theory of operator algebras" (Acad. Press: New York, 1983).

[13] I.L. Kantor, A.S. Solodovnikov. "Hypercomplex numbers" ( SpringerVerlag: Berlin, 1989).

[14] R. Killip, B. Simon. "Sum rules and spectral measures of Schrödinger operators with $L^{2}$ potentials". Annals of Mathematics 170: 2 (2009), 739-782. 
[15] R.S. Krausshar, J. Ryan. "Some conformally flat spin manifolds, Dirac operators and automorphic forms". J. Math. Anal. Appl. 325 (2007), 359-376.

[16] V.V. Kravchenko. "On a new approach for solving Dirac equations with some potentials and Maxwell's sytem in inhomogeoneous media". Operator Theory 121 (2001), 278-306.

[17] K. Kuratowski. "Topology" (Mir: Moscow, 1966).

[18] S.V. Ludkovsky, F. van Oystaeyen. "Differentiable functions of quaternion variables". Bull. Sci. Math. (Paris). Ser. 2.127 (2003), 755-796.

[19] S.V. Ludkovsky. "Differentiable functions of Cayley-Dickson numbers and line integration". J. of Mathem. Sciences 141: 3 (2007), 1231-1298.

[20] S.V. Ludkovsky. "Algebras of operators in Banach spaces over the quaternion skew field and the octonion algebra". J. Mathem. Sciences 144: 4 (2008), 4301-4366.

[21] S.V. Ludkovsky. "Residues of functions of octonion variables". Far East Journal of Mathematical Sciences (FJMS), 39: 1 (2010), 65-104.

[22] S.V. Ludkovsky. " Analysis over Cayley-Dickson numbers and its applications" (LAP Lambert Academic Publishing: Saarbrücken, 2010).

[23] S.V. Ludkovsky, W. Sproessig. "Ordered representations of normal and super-differential operators in quaternion and octonion Hilbert spaces". Adv. Appl. Clifford Alg. 20: 2 (2010), 321-342.

[24] S.V. Ludkovsky, W. Sprössig. "Spectral theory of super-differential operators of quaternion and octonion variables", Adv. Appl. Clifford Alg. 21: 1 (2011), 165-191.

[25] S.V. Ludkovsky, W. Sprössig. "Spectral representations of operators in Hilbert spaces over quaternions and octonions", Complex Variables and Elliptic Equations, online, DOI:10.1080/17476933.2010.538845, 24 pages (2011). 
[26] S.V. Ludkovsky. "Integration of vector hydrodynamical partial differential equations over octonions". Complex Variables and Elliptic Equations, online, DOI:10.1080/17476933.2011.598930, 31 pages (2011).

[27] S.V. Ludkovsky. "Line integration of Dirac operators over octonions and Cayley-Dickson algebras". Computational Methods and Function Theory, 12: 1 (2012), 279-306.

[28] S.V. Ludkovsky. "Operator algebras over Cayley-Dickson numbers" (LAP LAMBERT Academic Publishing AG \& Co. KG: Saarbrücken, 2011).

[29] S.V. Ludkovsky. "Unbounded normal operators in octonion Hilbert spaces and their spectra", Los Alamos Nat. Lab., math.FA/1204.1554 (2012), 49 pages.

[30] F. van Oystaeyen. "Algebraic geometry for associative algebras". Series "Lect. Notes in Pure and Appl. Mathem." 232 (Marcel Dekker: New York, 2000).

[31] R.D. Schafer. "An introduction to non-associative algebras" (Academic Press: New York, 1966).

[32] S. Zelditch. "Inverse spectral problem for analytic domains, II: $\mathbf{Z}_{2^{-}}$ symmetric domains". Advances in Mathematics 170: 1 (2009), 205269. 